\title{
The protective effect of umbelliferone ameliorates myocardial injury following ischemia-reperfusion in the rat through suppression NLRP3 inflammasome and upregulating the PPAR- $\gamma$
}

\author{
HONGBO LUO ${ }^{1 *}$, ZHENGKE FAN $^{2 *}$, DAOKANG XIANG ${ }^{1}$, ZHENWEI JIANG ${ }^{1}$, \\ WENBIN ZHANG $^{1}$, LUFANG GAO ${ }^{1}$ and CHAO FENG $^{1}$ \\ ${ }^{1}$ Department of Cardiovascular Surgery, The People's Hospital of Guizhou, Guiyang, Guizhou 550002; \\ ${ }^{2}$ Department of Cardiothoracic Surgery, The First People's Hospital, Bijie, Guizhou 551700, P.R. China
}

Received March 12, 2017; Accepted October 2, 2017

DOI: $10.3892 / \mathrm{mmr} .2017 .8208$

\begin{abstract}
The present study investigated whether the protective effect of umbelliferone could regulate myocardial injury following ischemia-reperfusion and improve mitochondrial respiratory function, thereby relieving myocardial injury following ischemia-reperfusion in rats. In the present study, the extent of inflammation and oxidative stress were analyzed using ELISA. Western blot analysis was employed to investigate the protein expression levels of the PYD domains-containing protein 3 (NLRP3) inflammasome and peroxisome proliferator-activated receptor- $\gamma$ $($ PPAR- $\gamma)$. Compared with the myocardial injury following ischemia-reperfusion group, umbelliferone significantly prevented myocardial injury, inhibited oxidative stress markers (superoxide dismutase and malondialdehyde), reduced inflammation (tumor necrosis factor- $\alpha$ and interleukin-6) and myocardial apoptosis levels (caspase-3/9 and apoptosis regular B-cell lymphoma-2-associated X protein) in the myocardial injury following ischemia-reperfusion group of rats. Umbelliferone treatment also suppressed NACHT, LRR and NLRP3 inflammasome activation and induced PPAR- $\gamma$ expression. The results of the present study suggested that the protective effect of umbelliferone may ameliorate myocardial injury following ischemia-reperfusion in the rat through the suppression of the NLRP3 inflammasome and upregulating PPAR- $\gamma$ expression.
\end{abstract}

Correspondence to: Dr Hongbo Luo, Department of Cardiovascular Surgery, The People's Hospital of Guizhou, 1 Baoshangnan Road, Guiyang, Guizhou 550002, P.R. China

E-mail: luohongbomm@sina.com

*Contributed equally

Key words: umbelliferone, inflammasome, peroxisome proliferatoractivated receptor- $\gamma$, myocardial injury, ischemia-reperfusion

\section{Introduction}

Myocardial ischemia/reperfusion (MI/R) injury was formerly referred to as myocardial infarction, as it is a disease that is caused by a sudden complete interruption of part of the myocardial blood circulation, leading to myocardial necrosis at that site (1). MI/R has become the primary cause of mortality in China, as the morbidity and mortality from $\mathrm{MI} / \mathrm{R}$ increases each year (2). The major cause of mortality in acute MI/R is acute heart failure (2). With the development of modern medicine, measures including internal medicine thrombolysis, surgical bypass and interventional stent surgery, have reduced the probability of mortality caused by the development of acute MI/R into acute heart failure (3). However, MI/R may enter a chronic phase as the disease develops further, which is dominated by myocardial fibrotic remodeling; excessive remodeling with fibrosis will induce chronic heart failure, therefore leading to the patient succumbing to heart failure (4).

Treatment of cardiovascular disease is based on inflammation control (5). The following aspects are essential in order to obtain the optimal anti-inflammatory therapeutic effects: First, excellent detection methods should be established in order to analyze adenosine triphosphate (ATP) ase activity in NOD-like receptor molecules and the issue of whether NACHT, LRR, and PYD domains-containing protein 3 (NLRP3) in myocardial tissue possesses tissue specificity should also be discussed, in order to apply the appropriate targeted therapy (6). Secondly, the downstream signaling mechanism following the binding of a ligand with the receptor should be further elucidated; excluding interleukin (IL)-1 $\beta$ and IL-18, NLRP3 can still activate other cytokines and inflammatory factors. There are a number of factors that inhibit NLRP3 in vivo, such as signaling stimulating the downregulation of Toll-like receptors (TLRs) and the release of the autologous IL-1 receptor antagonist (5). In addition, the IL-1 receptor antagonist has been reported to predict disease progression more effectively than IL-1 $\beta$ (6). Once these aforementioned issues have been resolved, more effective therapeutic strategies with higher selectivity, improved regulatory effects and decreased side effects can be developed (7). 
Peroxisome proliferator-activated receptor- $\gamma 1$ (PPAR- $\gamma 1)$ is a type of transcription factor activated by ligand engagement, and its activation serves an important role in regulating multiple pathophysiological processes (8). It has been reported that the administration of a PPAR- $\gamma$ ligand, such as pioglitazone, demonstrated protective effects on the I/R myocardium, the mechanism of which involved the increased expression of components of the signaling pathways associated with anti-oxidative stress, as well as the inhibition of inflammatory factor expression; however, each ligand was not associated with strong specificity and PPAR- $\gamma 2$, or other sub-types of PPAR, may frequently be activated during PPAR- $\gamma 1$ activation as well $(8,9)$.

Umbelliferone (structural formula exhibited in Fig. 1), a type of coumarin compound with the chemical name 7-hydroxycoumarin, is the major active ingredient of Rutaceae and Umbelliferae in Chinese herbal medicine, and belongs to the phenol family in terms of structure (10). Umbelliferone possesses anti-bacterial, anti-inflammatory and anti-apoptotic effects, which have been demonstrated in multiple Chinese herbal medicinal plants including Chinese Stellera chamaejasme Root, Radix Angelicae Pubescentis, Saussurea involucrata and Aegle marmelos $(11,12)$. In the present study, it was hypothesized that umbelliferone may protect cardiac tissues against MI/R via the modulation of the inflammasome and PPAR- $\gamma$.

\section{Materials and methods}

Animals and MI/R protocol. Adult male Sprague-Dawley rats (6-7 weeks, $n=24)$ with a body weight of 200-230 g were provided by the Laboratory Animal Center of Guizhou Medical University (Guizhou, China), housed at $22-23^{\circ} \mathrm{C}$, $55-60 \%$ atmosphere and maintained under a $12 \mathrm{~h}$ light/dark cycle with free access to food and water. The rats were randomly divided into the following 3 groups ( $\mathrm{n}=8 \mathrm{rats} / \mathrm{group}$ ): Control, MI/R model and the umbelliferone group. The rats were injected with sodium pentobarbital intraperitoneally (30 $\mathrm{mg} / \mathrm{kg}$ ) and the pericardium was dissected open. A left thoracic incision was conducted within rats of the MI/R and umbelliferone groups and a 6-0 silk suture slipknot was placed around the left anterior descending coronary artery to induce MI/R injury. Following $1 \mathrm{~h}$, the slipknot was released to re-perfuse the tissue. After $1 \mathrm{~h}$ following re-perfusion surgery, intraperitoneal injections of $30 \mathrm{mg} / \mathrm{kg} /$ day umbelliferone were administered for 7 days to the rats of the umbelliferone group. The rats of the control group were only injected with sodium pentobarbital $(30 \mathrm{mg} / \mathrm{kg})$ intraperitoneally and the pericardium was dissected open without MI/R injury as slipknot surgery was not performed. The protocol for the present study was approved by the Medical Ethics Committee of the People's Hospital of Guizhou Province (Guiyang, China).

Measurement of myocardial infarct size. Following treatment with umbelliferone and MI/R induction, rats were injected with $1 \mathrm{ml}$ of $2 \%$ Evans Blue dye into the aorta under sodium pentobarbital anesthesia $(30 \mathrm{mg} / \mathrm{kg}$, intraperitoneal injection) and immediately sacrificed. Heart tissues were then quickly frozen at $-70^{\circ} \mathrm{C}$ for $15 \mathrm{~min}$ and cut transversally

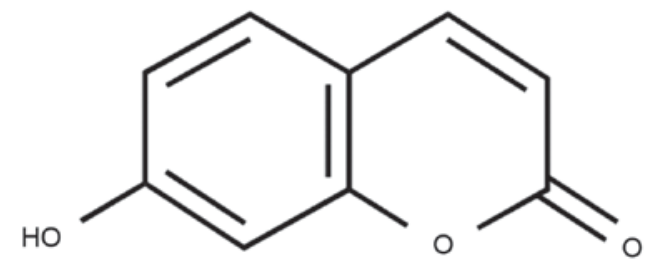

Figure 1. Structural formula of umbelliferone.

into 1-mm thick slices. Samples were incubated with 1\% 2,3,5-triphenyltetrazolium chloride (Sigma-Aldrich; Merck KGaA, Darmstadt, Germany) at $37^{\circ} \mathrm{C}$ for $10 \mathrm{~min}$ and observed with phase contrast microscopy (magnification, x10, Olympus IX83; Olympus, Tokyo, Japan).

Assessment of cardiac function. Heart tissues were homogenized and lysed with radioimmunoprecipitation assay (RIPA) buffer (Beyotime Institute of Biotechnology, Haimen, China) for $15 \mathrm{~min}$ at $4^{\circ} \mathrm{C}$ in the presence of protease inhibitors to extract protein. Protein content was isolated using a bicinchoninic acid (BCA) assay. A total of $5 \mu \mathrm{g}$ protein was used to measure creatine kinase (CK; A032), CK-muscle and brain (MB; H197) and lactate dehydrogenase (LDH; A020-2) activity using ELISA kits (Nanjing Jiangcheng Bioengineering Institute, Nanjing, China).

Determination of myocardial apoptosis, inflammation, oxidative stress and inducible nitric oxide synthase (iNOS) levels. Serum was collected following centrifugation at $1,000 \mathrm{x}$ g for $10 \mathrm{~min}$ at $4^{\circ} \mathrm{C}$ and was used to measure tumor necrosis factor- $\alpha$ (TNF- $\alpha$; PT516), IL-6 (PI328), IL-1 $\beta$ (PI303) (all from Beyotime Institute of Biotechnology) and IL-18 (H015; Nanjing Jiancheng Biology Engineering Institute, Nanjing, China) levels using ELISA kits. Heart tissues were homogenized and lysed with RIPA buffer (Beyotime Institute of Biotechnology) in the presence of protease inhibitors to extract protein. Protein content was isolated using a BCA assay. A total of $5 \mu \mathrm{g}$ protein was used to measure superoxide dismutase (SOD; S0101), malondialdehyde (MDA; S0131), caspase-3 (C1116), caspase-9 (AC062) and iNOS (S0025) (all from Beyotime Institute of Biotechnology) levels using ELISA kits.

Western blot analysis. Heart tissues were homogenized and lysed with RIPA assay (Beyotime Institute of Biotechnology) in the presence of protease inhibitors (PMSF, 1:100; Beyotime Institute of Biotechnology) to extract protein. Protein content was determined using a BCA assay and $50 \mu \mathrm{g}$ protein from each sample were loaded on to 2-3\% SDS-PAGE. Proteins were transferred to polyvinylidene difluoride membranes, which were blocked with $5 \%$ non-fat milk in Tris-buffered saline with $0.1 \%$ Tween- 20 for $1 \mathrm{~h}$ at $37^{\circ} \mathrm{C}$. Membranes were then incubated with primary antibodies against PPAR- $\gamma$ (1:500; sc-7196), B-cell lymphoma-2-associated X protein (Bax; 1:500; sc-6236), NLRP3 (1:500; sc-66846), caspase-1 (1:500; sc-514) and GAPDH (1:500; sc-25778) (all from Santa Cruz Biotechnology, Inc., Dallas, TX, USA) at $4^{\circ} \mathrm{C}$ overnight. The next day, blots were washed with TBST three times for 15 min and incubated with an anti-rabbit immunoglobulin $\mathrm{G}$ 

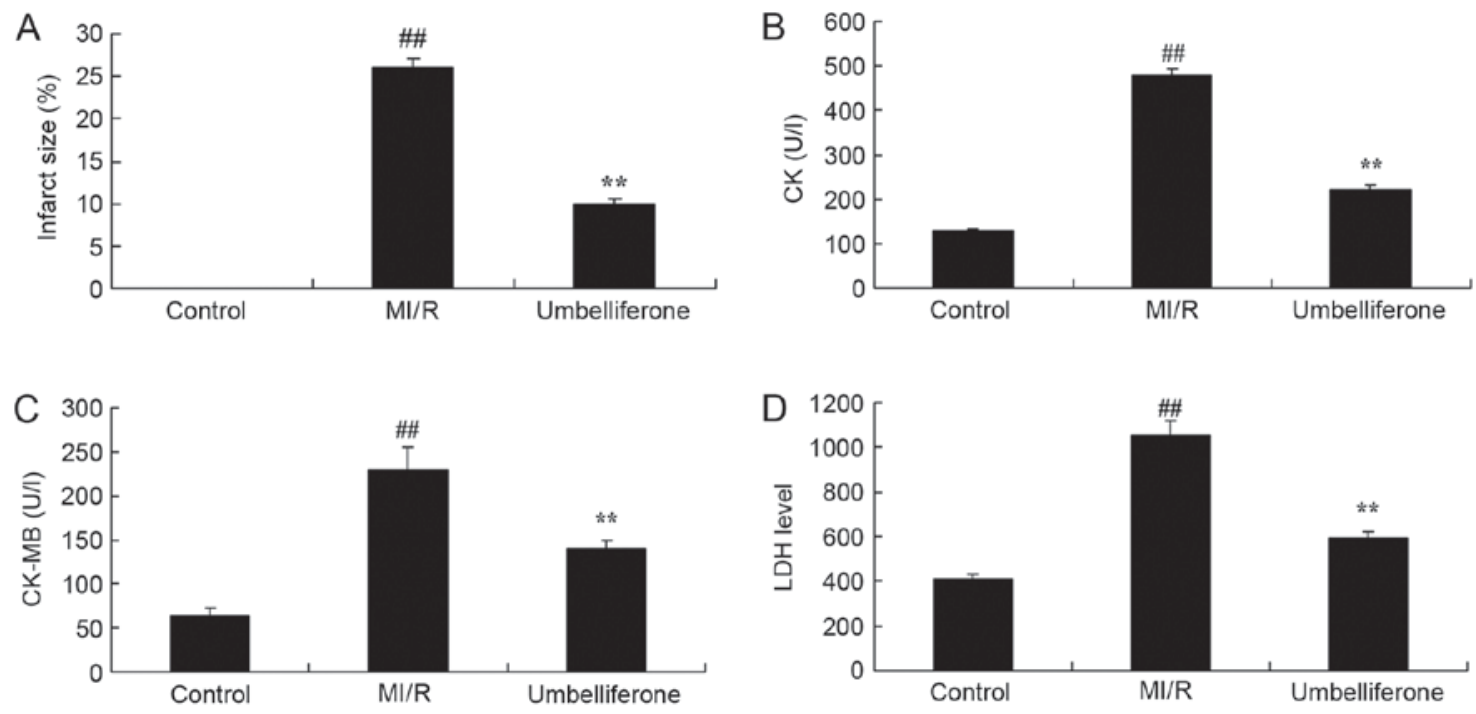

Figure 2. Umbelliferone significantly prevents myocardial injury. (A) Myocardial infarct size, and (B) CK, (C) CK-MB and (D) LDH activity levels in the control, MI/R and umbelliferone groups. The umbelliferone group was treated with $30 \mathrm{mg} / \mathrm{kg}$ umbelliferone. ${ }^{\# /} \mathrm{P}<0.01 \mathrm{vs}$. control group; ${ }^{* *} \mathrm{P}<0.01 \mathrm{vs}$. MI/R model group. CK-MB, creatine kinase-muscle and brain; LDH, lactate dehydrogenase; MI/R, myocardial ischemia/reperfusion.
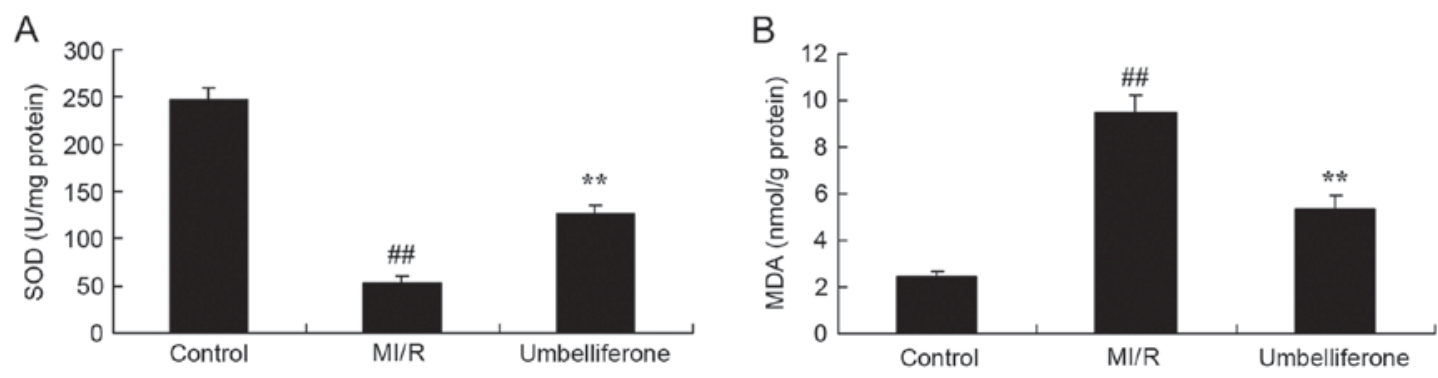

Figure 3. Umbelliferone significantly inhibited oxidative stress. Levels of (A) SOD and (B) MDA in the control, MI/R and umbelliferone groups. The umbelliferone group was treated with $30 \mathrm{mg} / \mathrm{kg}$ umbelliferone. ${ }^{\# /} \mathrm{P}<0.01$ vs. control group; ${ }^{* *} \mathrm{P}<0.01 \mathrm{vs}$. MI/R model group. MI/R, myocardial ischemia/reperfusion; SOD, superoxide dismutase; MDA, malondialdehyde.

(H+L) biotinylated secondary antibody $(1: 5,000,14708$; Cell Signaling Technology, Inc.) for $1 \mathrm{~h}$ at room temperature. The results were normalized to those of GAPDH and observed using BeyoECL Star (Beyotime Institute of Biotechnology) and analyzed using Image Lab 3.0 (Bio-Rad Laboratories, Inc., Hercules, CA, USA).

Statistical analysis. The data are presented as the mean \pm standard error of the mean $(n=3)$ using SPSS 17.0 (SPSS Inc., Chicago, IL, USA). For more than two groups, one-way analysis of variance with a Bonferroni post hoc test was conducted. $\mathrm{P}<0.05$ was considered to indicate a statistically significant difference.

\section{Results}

Umbelliferone significantly prevents myocardial injury. The protective effect of umbelliferone on myocardial injury was evaluated, by measuring myocardial infarct size and cardiac function. As demonstrated in Fig. 2, there were significant increases in myocardial infarct size, and CK, CK-MB and LDH activity in the MI/R model group, when compared with the control group. However, treatment with umbelliferone significantly reduced myocardial infarct size, and CK, CK-MB and LDH activity in MI/R mice when compared with the MI/R group $(\mathrm{P}<0.01$; Fig. 2$)$.

Umbelliferone significantly inhibits oxidative stress. ELISA analysis demonstrated that oxidative stress was increased, and the SOD level was lower and MDA level was higher in the $\mathrm{MI} / \mathrm{R}$ model group compared with in the control group $(\mathrm{P}<0.01$; Fig. 3). Umbelliferone treatment significantly increased SOD level and inhibited MDA level in MI/R mice, compared with in the MI/R model group (Fig. 3).

Umbelliferone significantly inhibits inflammation. To determine the anti-inflammatory effects of umbelliferone in MI/R, TNF- $\alpha$ and IL- 6 levels were measured in mice treated with umbelliferone. As shown in Fig. 4, ELISA analysis demonstrated that there was a significant increase in TNF- $\alpha$ and IL-6 levels in the MI/R model group, compared with the control group. The increase in TNF- $\alpha$ and IL- 6 levels in MI/R rats was significantly inhibited by umbelliferone treatment $(\mathrm{P}<0.01$; Fig. 4).

Umbelliferone significantly inhibits myocardial caspase levels. To evaluate the anti-apoptotic effects of umbelliferone in MI/R, the activity of caspase-3 and -9 were measured in 

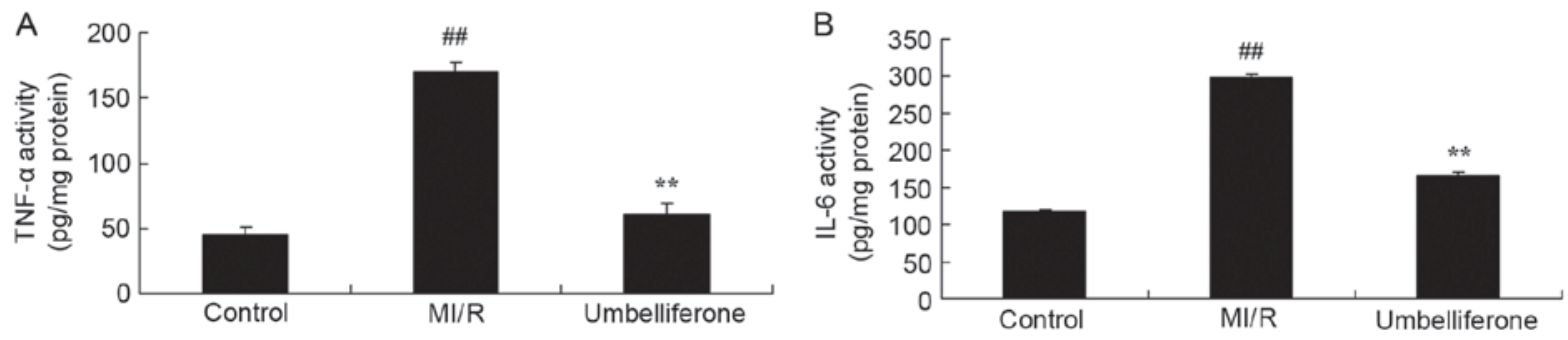

Figure 4. Umbelliferone significantly inhibited inflammatory markers. Levels of (A) TNF- $\alpha$ and (B) IL-6 in the control, MI/R and umbelliferone groups. The umbelliferone group was treated with $30 \mathrm{mg} / \mathrm{kg}$ umbelliferone. ${ }^{\# \#} \mathrm{P}<0.01 \mathrm{vs}$. control group; ${ }^{* * *} \mathrm{P}<0.01 \mathrm{vs}$. MI/R model group. MI/R, myocardial ischemia/reperfusion; TNF- $\alpha$, tumor necrosis factor- $\alpha$; IL-6, interleukin-6.
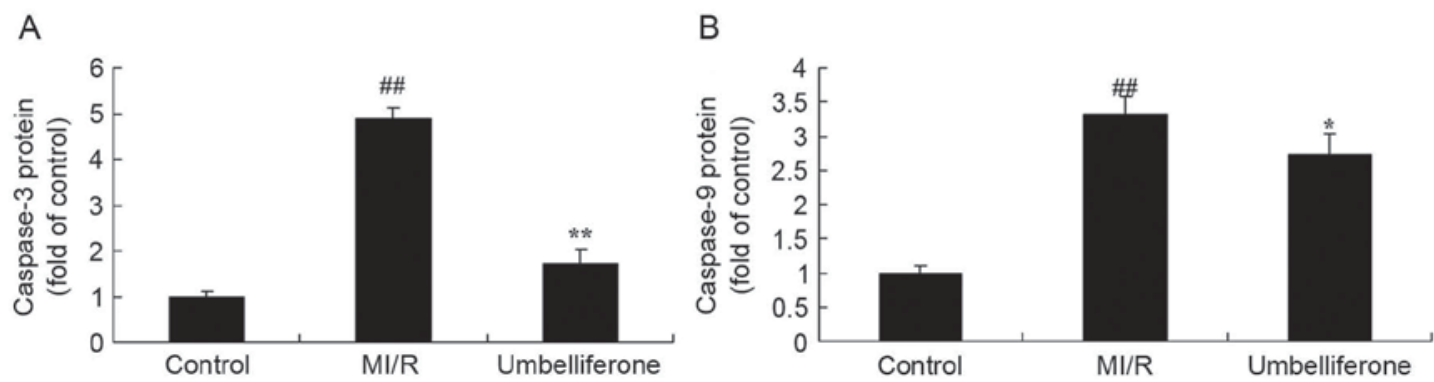

Figure 5. Umbelliferone significantly inhibited myocardial apoptosis markers. Levels of (A) caspase-3 and (B) caspase-9 activity in the control, MI/R and umbelliferone groups. The umbelliferone group was treated with $30 \mathrm{mg} / \mathrm{kg}$ umbelliferone. ${ }^{\# \#} \mathrm{P}<0.01$ vs. control group; ${ }^{*} \mathrm{P}<0.05 \mathrm{vs}$. MI/R model group. $\mathrm{MI} / \mathrm{R}$, myocardial ischemia/reperfusion.

A

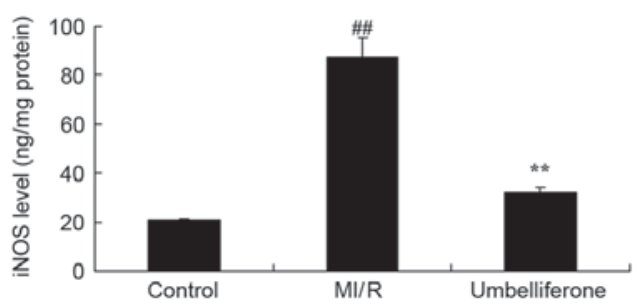

B

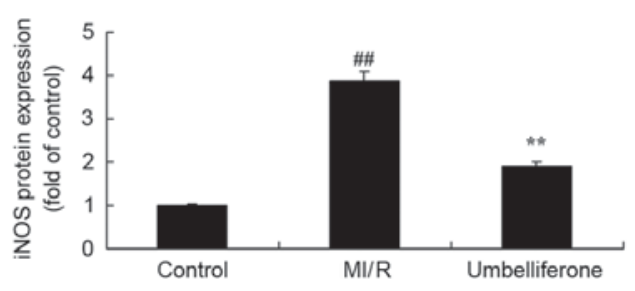

C

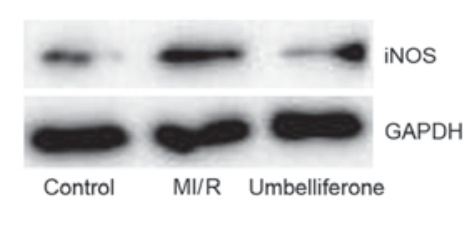

Figure 6. Umbelliferone significantly inhibited iNOS activity and protein expression. The (A) activity level, (B) quantified protein expression and (C) western blotting of iNOS in the control, MI/R and umbelliferone groups. The umbelliferone group was treated with $30 \mathrm{mg} / \mathrm{kg}$ umbelliferone. ${ }^{* \prime \prime} \mathrm{P}<0.01 \mathrm{vs}$. control group; ${ }^{* *} \mathrm{P}<0.01$ vs. MI/R model group. MI/R, myocardial ischemia/reperfusion; iNOS, inducible nitric oxide synthase.

$\mathrm{MI} / \mathrm{R}$ rats treated with umbelliferone. Caspase- 3 and -9 activity in the MI/R model group was significantly increased when compared with the control group $(\mathrm{P}<0.01$; Fig. 5). However, this increase in caspase -3 and -9 activity in MI/R rats was reduced by treatment with umbelliferone (Fig. 5).

Umbelliferone significantly inhibits iNOS activity and protein expression. To investigate the cardioprotective mechanism of umbelliferone in MI/R, alterations in iNOS activity and protein expression were analyzed. iNOS activity and protein expression were significantly increased in the MI/R model group when compared with the control group ( $\mathrm{P}<0.01$; Fig. 6). However, this increase in iNOS activity and protein expression in MI/R rats was significantly inhibited by treatment with umbelliferone ( $\mathrm{P}<0.01$; Fig. 6).

Umbelliferone significantly induces PPAR- $\gamma$ expression in rats with MI/R injury. Next, it was demonstrated that PPAR- $\gamma$ protein expression was downregulated and Bax protein expression was upregulated in the MI/R model group when compared with the control group (Fig. 7). Umbelliferone significantly induced PPAR- $\gamma$ protein expression and suppressed Bax protein expression when compared with the $\mathrm{MI} / \mathrm{R}$ rats $(\mathrm{P}<0.01 ;$ Fig. 7$)$.

Umbelliferone significantly suppresses NLRP3 inflammasome activation. To further investigate the anti-inflammatory effects of umbelliferone in MI/R, the activation of the NLRP3 inflammasome was analyzed. NLRP 3 and caspase-1 protein expression were significantly increased in the $\mathrm{MI} / \mathrm{R}$ model group when compared with the control group ( $<<0.01$; Fig. 8A-C). IL-1 $\beta$ and IL-18 activity levels were also increased in the MI/R model group when compared with the control group (Fig. 8D and E). However, umbelliferone significantly suppressed NLRP3 and caspase-1 protein expression, and IL-1 $\beta$ and IL-18 activity levels when compared with the MI/R model group ( $\mathrm{P}<0.01$; Fig. 8). 

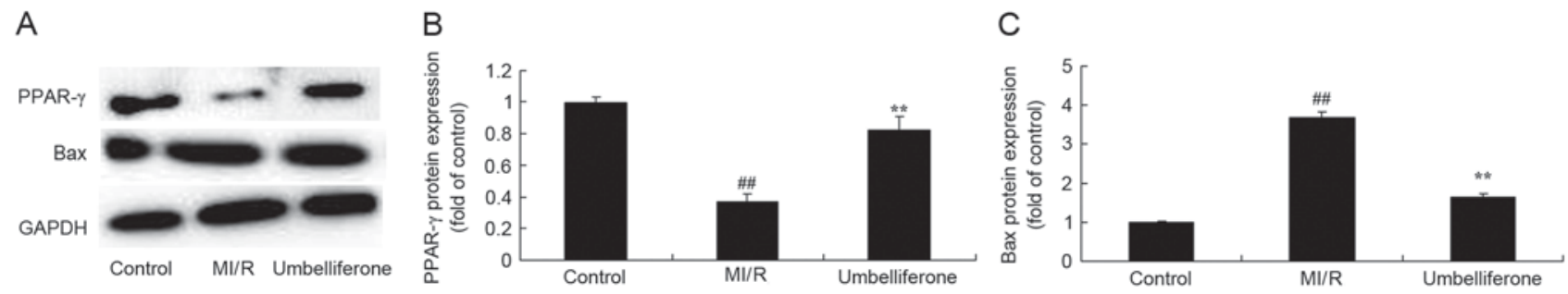

Figure 7. Umbelliferone significantly induced PPAR- $\gamma$ expression. PPAR- $\gamma$ and Bax protein expression using (A) western blotting, and statistical analysis of (B) PPAR- $\gamma$ and (C) Bax protein expression. The umbelliferone group was treated with $30 \mathrm{mg} / \mathrm{kg}$ umbelliferone. ${ }^{\# /} \mathrm{P}<0.01$ vs. control group; ${ }^{* *} \mathrm{P}<0.01 \mathrm{vs}$. MI/R model group. MI/R, myocardial ischemia/reperfusion; PPAR- $\gamma$, peroxisome proliferator-activated receptor- $\gamma$; Bax, B-cell lymphoma-2-associated X protein.

A

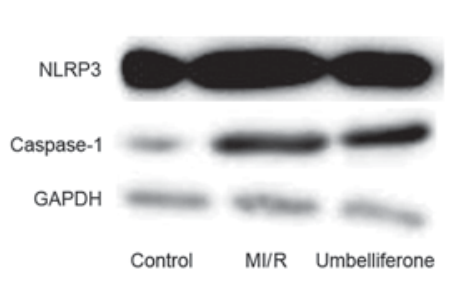

$\mathrm{B}$
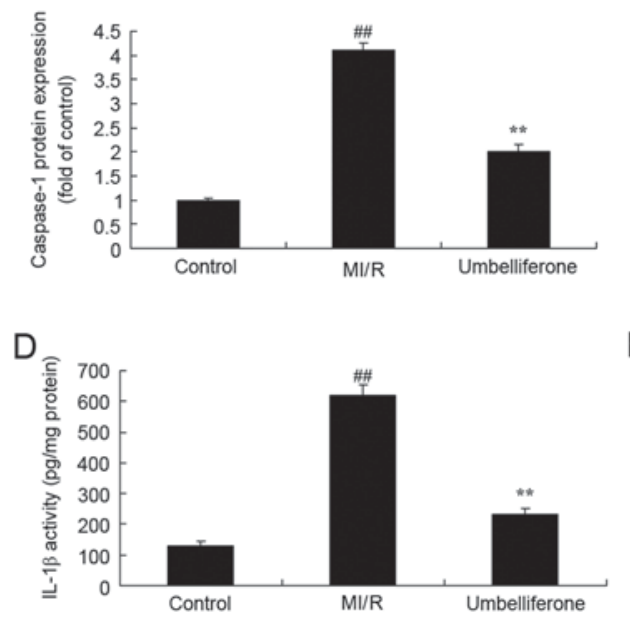

C

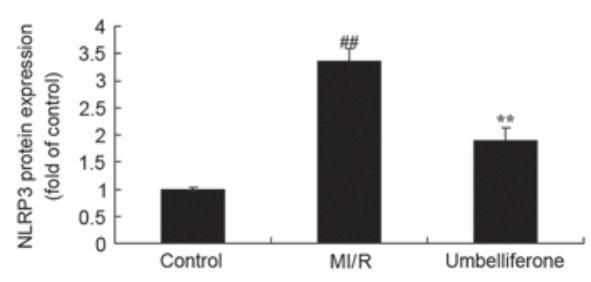

E

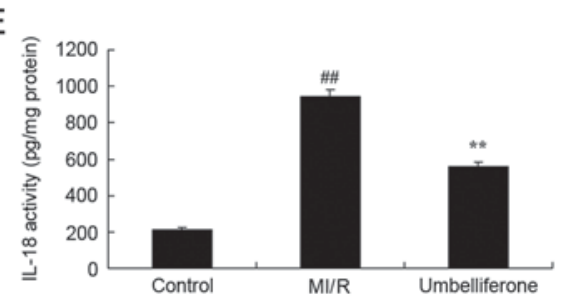

Figure 8. Umbelliferone significantly suppressed NLRP3 inflammasome activation. Investigation of NLRP3 and caspase-1 protein expression levels using (A) western blotting and statistical analysis of (B) caspase-1 and (C) NLRP3 protein expression, and (D) IL-1 $\beta$ and (E) IL-18 activity levels. The umbelliferone group was treated with $30 \mathrm{mg} / \mathrm{kg}$ umbelliferone. ${ }^{\# \#} \mathrm{P}<0.01 \mathrm{vs}$. control group; ${ }^{* *} \mathrm{P}<0.01 \mathrm{vs}$. MI/R model group. MI/R, myocardial ischemia/reperfusion; NLRP3, NACHT, LRR and PYD domains-containing protein 3; IL, interleukin.

\section{Discussion}

The levels of morbidity and mortality as a result of myocardial infarction (MI) in China are increasing every year and MI is also now affecting a younger demographic (13). At present, MI is primarily treated by drug and interventional therapy in clinical practice, which can partly alleviate symptoms and improve patient quality of life; however, it cannot fundamentally repair or reconstruct the injured or infarcted myocardium, which gives rise to the high mortality rates associated with MI (13). Cell therapy strategies have been previously developed, that can repair the injured myocardium to some extent; however, they cannot improve the hostile MI microenvironment (including ischemia, hypoxia, apoptosis and inflammation), and therefore, produce low retention and survival rates in the MI region, as well as unsatisfying long-term effects (14). With the further developments made in material science, life sciences, medical science and engineering, the injectable myocardial tissue engineering strategy with the basic elements of biomaterials, seeding cells and growth factors is promising to overcome the drawbacks of cell transplantation strategy, with an aim to realize myocardial repair and reconstruction in a real sense; it has subsequently become a key area of interest for research into treatments for MI (15). The results of the present study demonstrated that treatment with umbelliferone significantly reduced myocardial infarct size, and $\mathrm{CK}, \mathrm{CK}-\mathrm{MB}$ and $\mathrm{LDH}$ activity in an $\mathrm{MI} / \mathrm{R}$ rat model.

A previous study indicated that MI leads to massive necrosis and dissolution of myocardial cells, as well as inflammatory cell infiltration, thereby resulting in decreased cardiac function (16). MI produces an abnormal intracellular environment as a result of insufficient energy supply, and the compensatory heart contraction will give rise to an elevated level of reactive oxygen species (ROS) induced by nicotinamide adenine dinucleotide phosphate $(17,18)$. An elevated ROS level will in turn trigger a massive production of ROS by the mitochondria (16). Oxidative stress causes extensive damage to cell membranes and organelles, and can also induce an inflammatory response through mutual enhancement with inflammatory factors, further aggravating MI-induced myocardial injury (19). Consequently, improving the antioxidant enzyme level and reducing ROS content are some of the main objectives of developing treatments for MI (19). In the present study it was demonstrated that umbelliferone treatment significantly inhibited SOD levels and increased MDA levels in MI/R rats. Vijayalakshmi et al (12) suggested that umbelliferone prevented the incidence of tumors in 7,12-dimethylbenz[a]anthracene-induced oral carcinogenesis via antioxidant and xenobiotic metabolic effects. 
The inflammatory response exhibits bidirectional effects during the MI and post-MI repair phases; the early inflammatory response can eliminate necrotic tissue, promote granulation tissue formation, restrict injury of ischemia to the MI surrounding tissues, stabilize the extracellular matrix and promote scar formation (17). However, an excessive inflammatory response will induce tissue injury in the MI surrounding area, expand the MI size, enhance myocardial fibrosis, promote ventricular remodeling, reduce cardiac function and can lead to acute cardiac rupture (20). Furthermore, in the present study it was demonstrated that umbelliferone significantly reduced TNF- $\alpha$ and IL-6 levels in MI/R rats. Chunhua et al (11) reported that umbelliferone reverses depression-like behavior via the nuclear receptor-interacting protein 140/nuclear factor (NF) $-\kappa \mathrm{B}$ signaling pathway in chronic unpredictable mild stress-induced mice.

It has been demonstrated in a previous study that MI will induce an aseptic inflammatory response (21). The following phenomena supports the hypothesis that NLRP3 may be involved in this process (21). Expression of apoptosis-associated speck-like protein containing a CARD (ASC) and caspase-1 is increased in infiltrating macrophages and neutrophils in myocardial tissue following MI in rats with ASC-deletion; in addition, NLRP3 can identify multiple damage-associated molecular patterns (DAMPs) that are produced following myocardial injury (21). During the hepatic I/R process, NLRP3 promotes the release of inflammatory factors and leads to $\mathrm{I} / \mathrm{R}$ injury (21). I/R injury is reduced in NLRP3-deletion rat models (21). Sandanger et al (22) indicated that cardiac fibroblast may serve a role in initiating inflammation during the early stage of MI. A study proposed the following hypotheses: i) DAMPs may be released following injury in myocardial cells as a result of insufficient or interrupted blood supply, and TLRs on cardiac fibroblast membranes may identify DAMPs and promote the intracellular expression of $\mathrm{NF}-\kappa \mathrm{B}$ and NLRP3; ii) the injured cardiac fibroblast may develop ATP-dependent $\mathrm{K}^{+}$outflow, and a large amount of ROS may be produced during reperfusion, which may further activate NLRP3 in cardiac fibroblasts (5). This result demonstrated that umbelliferone significantly suppressed NLRP3 and caspase-1 protein expression in MI/R rats. Wang et al (23) suggested that umbelliferone may be protective against focal cerebral ischemia partly through the NLRP3 inflammasome and activation of PPAR- $\gamma$.

PPAR $-\gamma$ is a transcription factor in the nuclear receptor superfamily that is activated by a ligand (24). The activated ligand forms a heterodimer with the retinoic acid $\mathrm{X}$ receptor and then binds with the specific PPAR response element in the target gene promoter, thereby promoting or inhibiting the expression of its downstream gene, and exerting regulatory effects at the transcriptional level (24). Its activation is associated with multiple diseases, including diabetes, lipid metabolism, atherosclerosis and cancer (8). A previous study demonstrated that myocardial fibers are sparsely arranged in a disorderly manner, with a wide intercellular space and high levels of red blood cell and inflammatory cell infiltration in the stroma; while relatively ordered and densely arranged myocardial fibers can be seen in the PPAR- $\gamma$ group, with mild cell swelling, and little red blood cell and inflammatory cell infiltration (25). In the present study, it was demonstrated that umbelliferone significantly induced PPAR- $\gamma$ protein expression, and suppressed Bax and iNOS protein expression in the $\mathrm{MI} / \mathrm{R}$ rat model. Mahmoud et al (26) demonstrated that the protective effect of umbelliferone may prevent cyclophosphamide-induced hepatotoxicity through PPAR- $\gamma$ expression.

In conclusion, the results of the present study demonstrated that umbelliferone may ameliorate myocardial injury, inflammation, oxidative stress and apoptosis through the suppression of the NLRP3 inflammasome and upregulation of PPAR- $\gamma$ expression in rat. Therefore, umbelliferone demonstrates potential as a drug for the treatment of MI/R or other cardiovascular diseases.

\section{Acknowledgements}

The present study was supported by Qiankehe Lh Zi (grant no. 2016, 7181).

\section{References}

1. Prasad A, Gersh BJ, Mehran R, Brodie BR, Brener SJ, Dizon JM, Lansky AJ, Witzenbichler B, Kornowski R, Guagliumi G, et al: Effect of ischemia duration and door-to-balloon time on myocardial perfusion in ST-segment elevation myocardial infarction: An analysis from HORIZONS-AMI trial (harmonizing outcomes with revascularization and stents in acute myocardial infarction). JACC Cardiovasc Interv 8: 1966-1974, 2015.

2. Grygier M, Araszkiewicz A, Lesiak M, Janus M, Kowal J, Skorupski W, Pyda M, Mitkowski P and Grajek S: New method of intracoronary adenosine injection to prevent microvascular reperfusion injury in patients with acute myocardial infarction undergoing percutaneous coronary intervention. Am J Cardiol 107: 1131-1135, 2011

3. Woo JS, Kim W, Ha SJ, Kim JB, Kim SJ, Kim WS, Seon HJ and Kim KS: Cardioprotective effects of exenatide in patients with ST-segment-elevation myocardial infarction undergoing primary percutaneous coronary interventio: Results of exenatide myocardial protection in revascularization study. Arterioscler Thromb Vasc Biol 33: 2252-2260, 2013.

4. Hamarneh A, Sivaraman V, Bulluck H, Shanahan H, Kyle B, Ramlall M, Chung R, Jarvis C, Xenou M, Ariti C, et al: The effect of remote ischemic conditioning and glyceryl trinitrate on perioperative myocardial injury in cardiac bypass surgery patients: Rationale and design of the ERIC-GTN study. Clin Cardiol 38: 641-646, 2015.

5. Toldo S, Marchetti C, Mauro AG, Chojnacki J, Mezzaroma E, Carbone S, Zhang S, Van Tassell B, Salloum FN and Abbate A: Inhibition of the NLRP3 inflammasome limits the inflammatory injury following myocardial ischemia-reperfusion in the mouse. Int J Cardiol 209: 215-220, 2016.

6. Yu SY, Tang L, Zhao GJ and Zhou SH: Statin protects the heart against ischemia-reperfusion injury via inhibition of the NLRP3 inflammasome. Int J Cardiol 229: 23-24, 2017.

7. Valle Raleigh J, Mauro AG, Devarakonda T, Marchetti C, He J, Kim E, Filippone S, Das A, Toldo S, Abbate A and Salloum FN: Reperfusion therapy with recombinant human relaxin-2 (Serelaxin) attenuates myocardial infarct size and NLRP3 inflammasome following ischemia/reperfusion injury via eNOS-dependent mechanism. Cardiovasc Res 113: 609-619, 2017.

8. Wu Y, Tan X, Tian J, Liu X, Wang Y, Zhao H, Yan Z, Liu H and Ma X: PPAR $\gamma$ agonist ameliorates the impaired fluidity of the myocardial cell membrane and cardiac injury in hypercholesterolemic rats. Cardiovasc Toxicol 17: 25-34, 2017.

9. Ravingerova T, Adameova A, Carnicka S, Nemcekova M, Kelly T, Matejikova J, Galatou E, Barlaka E and Lazou A: The role of PPAR in myocardial response to ischemia in normal and diseased heart. Gen Physiol Biophys 30: 329-341, 2011.

10. Rezaee R, Behravan E, Behravan J, Soltani F, Naderi Y, Emami B and Iranshahi M: Antigenotoxic activities of the natural dietary coumarins umbelliferone, herniarin and 7-isopentenyloxy coumarin on human lymphocytes exposed to oxidative stress. Drug Chem Toxicol 37: 144-148, 2014. 
11. Chunhua M, Lingdong K, Hongyan L and Zhangqiang $\mathrm{M}$ : Retracted: Umbelliferone reverses depression-like behavior in chronic unpredictable mild stress-induced mice via RIP140/NF-кB pathway. IUBMB Life 69: 767, 2017.

12. Vijayalakshmi A and Sindhu G: Dose responsive efficacy of umbelliferone on lipid peroxidation, anti-oxidant, and xenobiotic metabolism in DMBA-induced oral carcinogenesis. Biomed Pharmacother 88: 852-862, 2017.

13. Hofsten DE, Kelbaek H, Helqvist S, Kløvgaard L, Holmvang L, Clemmensen P, Torp-Pedersen C, Tilsted HH, Bøtker HE, Jensen LO, et al: The third DANish study of optimal acute treatment of patients with ST-segment elevation myocardial infarction: Ischemic postconditioning or deferred stent implantation versus conventional primary angioplasty and complete revascularization versus treatment of culprit lesion only: Rationale and design of the DANAMI 3 trial program. Am Heart J 169: 613-621, 2015.

14. Hausenloy DJ, Candilio L, Laing C, Kunst G, Pepper J, Kolvekar S, Evans R, Robertson S, Knight R, Ariti C, et al: Effect of remote ischemic preconditioning on clinical outcomes in patients undergoing coronary artery bypass graft surgery (ERICCA): Rationale and study design of a multi-centre randomized double-blinded controlled clinical trial. Clin Res Cardiol 101: 339-348, 2012.

15. Gu YL, Kampinga MA, Wieringa WG, Fokkema ML, Nijsten MW, Hillege HL, van den Heuvel AF, Tan ES Pundziute $\mathrm{G}$, van der Werf R, et al: Intracoronary versus intravenous administration of abciximab in patients with ST-segment elevation myocardial infarction undergoing primary percutaneous coronary intervention with thrombus aspiration: The comparison of intracoronary versus intravenous abciximab administration during emergency reperfusion of ST-segment elevation myocardial infarction (CICERO) trial. Circulation 122: 2709-2717, 2010

16. Pei H, Song X, Peng C, Tan Y,Li Y,Li X, Ma S, Wang Q, Huang R, Yang D, et al: TNF- $\alpha$ inhibitor protects against myocardial ischemia/reperfusion injury via Notch1-mediated suppression of oxidative/nitrative stress. Free Radic Biol Med 82: 114-121, 2015.

17. Yue R, Xia X, Jiang J, Yang D, Han Y, Chen X, Cai Y, Li L, Wang WE and Zeng C: Mitochondrial DNA oxidative damage contributes to cardiomyocyte ischemia/reperfusion-injury in rats: Cardioprotective role of lycopene. J Cell Physiol 230: 2128-2141, 2015.
18. Yu Z, Wang S, Zhang X, Li Y, Zhao Q and Liu T: Pterostilbene protects against myocardial ischemia/reperfusion injury via suppressing oxidative/nitrative stress and inflammatory response. Int Immunopharmacol 43: 7-15, 2017.

19. Zheng P, Xie Z, Yuan Y, Sui W, Wang C, Gao X, Zhao Y, Zhang F, $\mathrm{Gu} \mathrm{Y}, \mathrm{Hu} \mathrm{P}$, et al: Plin5 alleviates myocardial ischaemia/reperfusion injury by reducing oxidative stress through inhibiting the lipolysis of lipid droplets. Sci Rep 7: 42574, 2017.

20. Zhang J, Zhang J, Yu P, Chen M, Peng Q, Wang Z and Dong N: Remote ischaemic preconditioning and sevoflurane postconditioning synergistically protect rats from myocardial injury induced by ischemia and reperfusion partly via Inhibition TLR4/MyD88/NF- $\kappa$ B signaling pathway. Cell Physiol Biochem 41: 22-32, 2017.

21. Sandanger $\varnothing$, Ranheim T, Vinge LE, Bliksøen M, Valen G, Aukrust P and Yndestad A: A role for NLRP3 inflammasome in acute myocardial ischaemia-reperfusion injury? Reply. Cardiovasc Res 99: 226-227, 2013.

22. Sandanger Ø, Ranheim T, Vinge LE, Bliksøen M, Alfsnes K, Finsen AV, Dahl CP, Askevold ET, Florholmen G, Christensen G, et al: The NLRP3 inflammasome is up-regulated in cardiac fibroblasts and mediates myocardial ischaemia-reperfusion injury. Cardiovasc Res 99: 164-174, 2013.

23. Wang X, Li R, Wang X, Fu Q and Ma S: Umbelliferone ameliorates cerebral ischemia-reperfusion injury via upregulating the PPAR gamma expression and suppressing TXNIP/NLRP3 inflammasome. Neurosci Lett 600: 182-187, 2015.

24. Liu X, Yu Z, Huang X, Gao Y, Wang X, Gu J and Xue S: Peroxisome proliferator-activated receptor $\gamma(\operatorname{PPAR} \gamma)$ mediates the protective effect of quercetin against myocardial ischemia-reperfusion injury via suppressing the NF- $\kappa \mathrm{B}$ pathway. Am J Transl Res 8: 5169-5186, 2016.

25. Qian J, Chen H, Birnbaum Y, Nanhwan MK, Bajaj M and Ye Y: Aleglitazar, a balanced dual PPAR $\alpha$ and $-\gamma$ agonist, protects the heart against ischemia-reperfusion injury. Cardiovasc Drugs Ther 30: 129-141, 2016.

26. Mahmoud AM, Germoush MO, Alotaibi MF and Hussein OE: Possible involvement of Nrf2 and PPAR $\gamma$ up-regulation in the protective effect of umbelliferone against cyclophosphamide-induced hepatotoxicity. Biomed Pharmacother 86: 297-306, 2017. 